\title{
CORACH-PORTA-RECHT INEQUALITY
} FOR CLOSED RANGE OPERATORS

\section{MARYAM KHOSRAVI}

Abstract. By $\mathbb{B}(\mathscr{H})$ we denote the space of all bounded linear operators on a Hilbert space $\mathscr{H}$. In 2001, Seddik characterized all invertible self-adjoint operators using the Corach-Porta-Recht inequality

$$
\left\|S X S^{-1}+S^{-1} X S\right\| \geqslant 2\|X\| .
$$

In this paper, we find a characterization of closed range self-adjoint operators using a version of this inequality for closed range operators.

Mathematics subject classification (2010): Primary: 47A30, 15A09, secondary: 47B15.

Keywords and phrases: Moore-Penrose inverse, operator inequality, closed range operators, self-adjoint operators.

\section{Introduction and preliminaries}

In [1], Corach, Porta and Recht proved that if $S$ is a self-adjoint invertible operator on a Hilbert space $\mathscr{H}$, then for all $X \in \mathbb{B}(\mathscr{H})$ the following (C-P-R) inequality holds:

$$
\left\|S X S^{-1}+S^{-1} X S\right\| \geqslant 2\|X\| .
$$

They used this inequality as a key factor in their study of differential geometry. J. I. Fujii, M. Fujii, Furuta and Nakamoto [2], showed that this inequality is equivalent to Heinz inequality which is one of the most essential inequalities in operator theory.

Seddik [5] could find a characterization of non-zero scalars of invertible selfadjoint operators base on this inequality.

In this paper we discuss about a version of this inequality for Moore-Penrose invertible operators.

Definition 1.1. Let $\mathscr{A}$ be an algebra with involution and $a \in \mathscr{A}$. If there exists an element $x \in \mathscr{A}$ satisfied the following four equations

$$
\begin{array}{ll}
a x a=a & x a x=x \\
(a x)^{*}=a x & (x a)^{*}=x a,
\end{array}
$$

then $x$ is called a Moore-Penrose inverse of $a$ and denoted by $a^{\dagger}$.

It is easy to show that the Moore-Penrose inverse of an element $a$ is unique.

If $a \in \mathscr{A}$ is Moore-Penrose invertible, then

(a) $a^{\dagger \dagger}=a$, 
(b) $a^{*}$ is Moore-Penrose invertible and $a^{*^{\dagger}}=a^{\dagger^{*}}$,

(c) If $a$ is invertible, then $a^{\dagger}=a^{-1}$,

(d) $\left(a a^{*}\right)^{\dagger}=a^{*^{\dagger}} a^{\dagger}$.

Harte and Mbekhta in [3] proved that if $\mathscr{H}$ is a Hilbert space and $T \in \mathbb{B}(\mathscr{H})$ then the following conditions are equivalent:

i) $T$ has a generalized inverse (that is there exists an operator $S \in \mathbb{B}(\mathscr{H})$ for which $T S T=T$ and $S T S=S$ ).

ii) $\mathscr{R}(T)$ is closed.

iii) $T$ has a Moore-Penrose inverse.

In this case $T T^{\dagger}$ is the projection on $\mathscr{R}(T)$ and $T^{\dagger} T$ is the projection on $\mathscr{R}\left(T^{*}\right)$.

In this note, we present a version of C-P-R inequality for Moore-Penrose invertible operators. In addition, for a close subspace $\mathscr{K}$ of Hilbert space $\mathscr{H}$, we give a characterization of all hermitian operators with range $\mathscr{K}$.

\section{Main results}

In [4], McIntosh proved that

$$
\left\|A^{*} A X+X B B^{*}\right\| \geqslant 2\|A X B\|,
$$

for all operators $A, X, B \in \mathbb{B}(\mathscr{H})$. Using this inequality we can state the following result.

THEOREM 2.1. Let $S$ be a hermitian operator on Hilbert space $\mathscr{H}$ such that $\mathscr{R}(S)$ is closed. Then

$$
\left\|S X S^{\dagger}+S^{\dagger} X S\right\| \geqslant 2\|P X P\|,
$$

where $P=S S^{\dagger}$.

Proof. From McIntosh inequality and the relation $S S^{\dagger}=\left(S S^{\dagger}\right)^{*}=S^{\dagger} S$, we have

$$
\left\|S X S^{\dagger}+S^{\dagger} X S\right\|=\left\|S S\left(S^{\dagger} X S^{\dagger}\right)+\left(S^{\dagger} X S^{\dagger}\right) S S\right\| \geqslant 2\left\|S S^{\dagger} X S^{\dagger} S\right\|=2\|P X P\| .
$$

In general, it is not true that $(a b)^{\dagger}=b^{\dagger} a^{\dagger}$. However, in polar decomposition of an operator we can deduced the next result.

LEMMA 2.2. Let $S$ be an operator with close range and $S=U|S|$ be the polar decomposition of $S$. Then

$$
S^{\dagger}=|S|^{\dagger} U^{*}, \quad \& \quad|S|^{\dagger}=S^{\dagger} U .
$$


Proof. First note that as a result of polar decomposition, we have $\mathscr{R}(|S|)=\mathscr{R}\left(S^{*}\right)$ and therefore is closed. Since the Moore-Penrose inverse is unique, the following relations lead to the first equation:

1. $S\left(|S|^{\dagger} U^{*}\right) S=U|S|\left(|S|^{\dagger} U^{*}\right) S=U|S||S|^{\dagger}|S|=U|S|=S$.

2. $\left(|S|^{\dagger} U^{*}\right) S\left(|S|^{\dagger} U^{*}\right)=|S|^{\dagger}|S||S|^{\dagger} U^{*}=|S|^{\dagger} U^{*}$.

3. $S\left(|S|^{\dagger} U^{*}\right)=U|S||S|^{\dagger} U^{*}$ which is hermitian, because $|S||S|^{\dagger}$ is hermitian.

4. $\left(|S|^{\dagger} U^{*} S\right)=|S|^{\dagger}|S|$ which is hermitian.

The second equality is proved similarly.

REMARK 2.3. By the previous lemma it is seen that

$$
|S||S|^{\dagger}=|S|^{\dagger}|S|=S^{\dagger} U|S|=S^{\dagger} S \text {. }
$$

In addition

$$
|S|^{\dagger}(H)=|S|^{\dagger}|S|(H)=S^{\dagger} S(H)=S^{\dagger}(H)=S^{*}(H)=|S|(H) .
$$

So if $S=U|S|$ is the polar decomposition of $S$, then $U$ is isometry on $|S|^{\dagger}(H)$.

Using Lemma 2.2, we can deduce the following version of Theorem 2.1, similarly to [2]:

THEOREM 2.4. Let $S, T$ be operators on Hilbert space $\mathscr{H}$ such that $\mathscr{R}(S)$ and $\mathscr{R}(T)$ are closed. Then

$$
\left\|S^{*} X T^{\dagger}+S^{\dagger} X T^{*}\right\| \geqslant 2\|P X Q\|,
$$

where $P=S S^{\dagger}$ and $Q=T^{\dagger} T$.

Proof. First we proved the inequality for the case that $T=S$.

Let $S=U|S|$ be the polar decomposition of $S$. Then

$$
\begin{aligned}
\left\|S^{*} X S^{\dagger}+S^{\dagger} X S^{*}\right\| & =\left\||S| U^{*} X|S|^{\dagger} U^{*}+|S|^{\dagger} U^{*} X|S| U^{*}\right\| \\
& =\left\|U\left(|S|^{\dagger} X^{*} U|S|+|S| X^{*} U|S|^{\dagger}\right)\right\| \\
& =\left\||S|^{\dagger} X^{*} U|S|+|S| X^{*} U|S|^{\dagger}\right\| \\
& \geqslant 2\left\||S||S|^{\dagger} X^{*} U|S||S|^{\dagger}\right\| \\
& =2\left\||S||S|^{\dagger} U^{*} X|S||S|^{\dagger}\right\| \\
& =2\left\|U^{*} S S^{\dagger} X S^{\dagger} S\right\| \\
& =2\left\|S S^{\dagger} X S^{\dagger} S\right\|=2\|P X Q\| .
\end{aligned}
$$


Now let $S, T$ be two arbitrary operators for which $\mathscr{R}(S)$ and $\mathscr{R}(T)$ is closed. Using the previous part, for closed range operator $\left[\begin{array}{ll}S & 0 \\ 0 & T\end{array}\right]$ and all operators of the form $\left[\begin{array}{ll}0 & X \\ 0 & 0\end{array}\right]$ on the Hilbert space $\mathscr{H} \oplus \mathscr{H}$, we have

$$
\begin{gathered}
\left\|\left[\begin{array}{cc}
S^{*} & 0 \\
0 & T^{*}
\end{array}\right]\left[\begin{array}{ll}
0 & X \\
0 & 0
\end{array}\right]\left[\begin{array}{cc}
S^{\dagger} & 0 \\
0 & T^{\dagger}
\end{array}\right]+\left[\begin{array}{cc}
S^{\dagger} & 0 \\
0 & T^{\dagger}
\end{array}\right]\left[\begin{array}{cc}
0 & X \\
0 & 0
\end{array}\right]\left[\begin{array}{cc}
S^{*} & 0 \\
0 & T^{*}
\end{array}\right]\right\| \\
\geqslant 2\left\|\left[\begin{array}{cc}
S S^{\dagger} & 0 \\
0 & T T^{\dagger}
\end{array}\right]\right\|\left[\begin{array}{cc}
0 & X \\
0 & 0
\end{array}\right]\left[\begin{array}{cc}
S^{\dagger} S & 0 \\
0 & T^{\dagger} T
\end{array}\right] \|,
\end{gathered}
$$

That is

$$
\left\|S^{*} X T^{\dagger}+S^{\dagger} X T^{*}\right\| \geqslant 2\|P X Q\| .
$$

In [5], Seddik obtained the following characterization of the invertible operators which satisfy the C-P-R inequality for all $X \in \mathbb{B}(\mathscr{H})$.

THEOREM 2.5. [5] The set of all invertible operators $S$, for which

$$
\forall X \in \mathscr{H},\left\|S X S^{-1}+S^{-1} X S\right\| \geqslant 2\|X\| .
$$

is the set $\left\{\lambda M: \lambda \in \mathbb{C}^{*}, M\right.$ is an invertible self-adjoint operator $\}$.

Now we prove a version of this theorem for Moore-Penrose invertible operators.

THEOREM 2.6. Let $\mathscr{K}$ be a closed subspace of $\mathscr{H}$ and $P$ be the projection on $\mathscr{K}$. If $S \in \mathbb{B}(\mathscr{H})$ with $\mathscr{R}(S)=\mathscr{R}\left(S^{*}\right)=\mathscr{K}$ and

$$
\forall X \in \mathbb{B}(\mathscr{H}),\left\|S X S^{\dagger}+S^{\dagger} X S\right\| \geqslant 2\|P X P\|,
$$

then $S=\lambda T$, for some non-zero $\lambda \in \mathbb{C}$ and some self-adjoint operator $T$ with $\mathscr{R}(T)=$ $\mathscr{K}$.

Proof. From the hypothesis, we can simply write

$$
\left\|S(P X P) S^{\dagger}+S^{\dagger}(P X P) S\right\|=\left\|S X S^{\dagger}+S^{\dagger} X S\right\| \geqslant 2\|P X P\|,
$$

So from Theorem 2.5, we have $S=\lambda T$ as operators on $\mathbb{B}(\mathscr{K})$. Since $S=0$ on $\mathscr{K}^{\perp}$, we can get the result.

From Theorem 2.1 and 2.6, the following theorem is immediately follows.

THEOREM 2.7. Let $\mathscr{K}$ be a closed subspace of $\mathscr{H}$ and $P$ be the projection on $\mathscr{K}$. If $S \in \mathbb{B}(\mathscr{H})$ with $\mathscr{R}(S)=\mathscr{R}\left(S^{*}\right)=\mathscr{K}$, then the following conditions are equivalent:

- $\forall X \in \mathbb{B}(\mathscr{H}),\left\|S X S^{\dagger}+S^{\dagger} X S\right\| \geqslant 2\|P X P\|$,

- $S=\lambda T$ for some self-adjoint operator $T$ with $\mathscr{R}(T)=\mathscr{K}$, and non-zero scalar $\lambda$. 


\section{Acknowledgment}

The author would like to express her thanks to the referee for useful and heartwarming suggestions.

\section{REFERENCES}

[1] G. Corach, R. Porta And L. Recht, An operator inequality, Linear Algebra Appl. 142 (1990), $153-158$.

[2] J. I. FujiI, M. FujiI, T. Furuta AND R. NAKAmoto, Norm inequalities equivalent to Heinz inequality, Proc. Amer. Math. Soc. 118 (1993), 827-830.

[3] R. Harte And M. Mbekhta, On generalized inverses in $C^{*}$-algebras, Studia Mathematics (1992), no. $103,71-77$.

[4] A. McIntosh, Heinz inequalities and perturbation of spectral families, Macquarie Mathematical Reports, Macquarie Univ., 1979.

[5] A. SEDdik, Some results related to the Corach-Porta-Recht inequality, Proc. Amer. Math. Soc. 129, 10 (1987), 3009-3015. 